\title{
Biological intratumoral therapy for the high-grade glioma part I: intratumoral delivery and immunotoxins
}

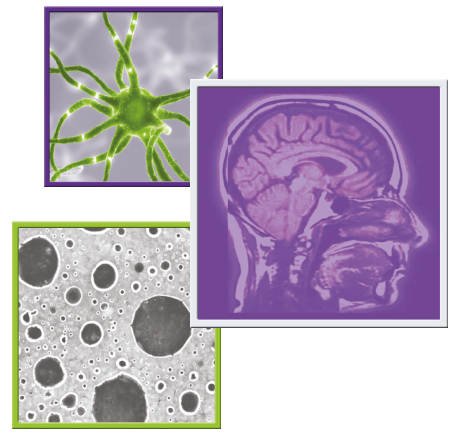

\author{
Joshua Loya ${ }^{1}$, Charlie Zhang ${ }^{2}$, Emily Cox ${ }^{3}$, Achal S Achrol*, $\ddagger 4$ \& Santosh Kesari**, $\ddagger, 4$ \\ ${ }^{1}$ Wayne State University School of Medicine, Department of Neurosurgery, 42 W Warren Ave, Detroit, MI 48202, USA \\ 2State University of New York at Buffalo School of Medicine, 1010 Main St, Buffalo, NY 14202, USA \\ ${ }^{3}$ Providence Medical Research Center, 105 W 8th Ave \#6050w, Spokane, WA 99204, USA \\ ${ }^{4}$ John Wayne Cancer Institute \& Pacific Neuroscience Institute, 2200 Santa Monica Blvd, Santa Monica, CA 90404, USA \\ *Author for correspondence: Tel.: +1 310829 8265; Fax: +1 310582 7287; achrol@jwci.org \\ **Author for correspondence: kesaris@jwci.org \\ $\ddagger$ Authors contributed equally
}

Management of high-grade gliomas remains a complex challenge. Standard of care consists of microsurgical resection, chemotherapy and radiation, but despite these aggressive multimodality therapies the overall prognosis remains poor. A major focus of ongoing translational research studies is to develop novel therapeutic strategies that can maximize tumor cell eradication while minimizing collateral side effects. Particularly, biological intratumoral therapies have been the focus of new translational research efforts due to their inherent potential to be both dynamically adaptive and target specific. This two-part review will provide an overview of biological intratumoral therapies and summarize key advances and remaining challenges in intratumoral biological therapies for high-grade glioma. Part I focuses on discussion of the concepts of intratumoral delivery and immunotoxin therapies.

First draft submitted: 12 January 2019; Accepted for publication: 19 June 2019; Published online: 21 November 2019

Keywords: biologic therapy $\bullet$ brain neoplasm • chemotherapy $\bullet$ drug delivery $\bullet$ glioblastoma $\bullet$ high-grade gliomas - immunotoxin • intratumoral delivery

High-grade gliomas (HGGs) remain the most challenging primary brain neoplasms to treat and necessitate a multimodal therapeutic approach. With an incidence of 5 per 100,000 they are the most common malignant brain neoplasm, and despite advancements in treatment over the last several decades, mortality remains high with a 1-year survival of $35.7 \%$ for glioblastoma and ranging from 60 to $80 \%$ for WHO grade III tumors [1]. There also exists a significant economic burden associated with HGG, with treatment costs estimated at over US $\$ 1.3$ billion annually in the USA [2].

The invasive nature of these lesions was recognized early by pioneers of neurosurgery, which was quickly found to intrinsically limit the efficacy of surgical resection alone in the management of this disease [3]. Intratumoral treatments developed as a result of the intuitive hypothesis that local administration of tumoricidal treatment may be more potent, a concept which grew in credence with advancements in our understanding of the blood-brain barrier (BBB), which is modified to form a blood-tumor barrier that prevents the delivery of systemic therapeutics to the target tumor and increases the expression of efflux pumps to diminish drug levels at the target site $[4,5]$. Therefore, in addition to the development and implementation of adjuvant therapies such as systemic chemotherapy and radiation therapy, there remained an early interest in surgically delivered therapies.

The use of biological agents in the treatment of HGG began in the 1970s when, stemming from the discovery of the immune system's significant role in other malignancies $[6,7,8]$, it was observed that lymphocytic infiltrates were present within the glioma on autopsy specimens in over a third of patients [9] and that the presence of these infiltrates correlated with improved survival [10-12]. Some of the earliest reported trials to use intratumorally administered biological therapeutics in HGG used autologous unsegregated lymphocytes [13], mononuclear cells [14], lymphokineactivated killer cells, IL-2 [15], and other agents aimed at supporting, activating, or otherwise targeting the immune

Future Medicine 
system against remnant tumor cells. Since these initial pioneering efforts at harnessing locally delivered biologically active agents to combat HGG, the realm of intratumoral biological therapeutics has grown and developed to include a wide breadth of modern advanced immune-based therapies and targeted gene therapies.

The goal of this two-part review is to summarize, discuss and elucidate the current and relevant biological intratumoral therapies being utilized in clinical trials in the treatment of HGGs. Biological therapies are defined as treatments whose mechanism is primarily based on an active cellular response or utilization of a biologically active agent to produce its antineoplastic effect. The present article (part I) will focus on the evolving concept and challenges of intratumoral therapeutics and a detailed review of immunotoxin therapy.

\section{Intratumoral therapeutic delivery}

The BBB is a selective barrier formed by endothelial cells joined by complex tight junctions that line cerebral microvessels preventing intercellular movement of most molecules [16]. The BBB normally serves to help maintain precise regulation of the physiologic microenvironment for the brain and preserve reliable neuronal signaling. However, in the context of a malignant glioma or other invasive neoplasm, it can serve as a hindrance preventing systemic chemotherapeutics from effectively reaching target cancer cells in the brain $[5,17]$. Intratumoral therapeutic delivery holds promise as an approach that may bypass the limitations posed by the BBB and could potentially provide a means to achieve greater concentrations and improved distribution of a therapeutic agent within target brain regions than could be achieved by systemic administration alone.

A variety of delivery methods for intratumoral therapeutics have been used in clinical trials for HGGs, including simple local injection into the resection cavity or bordering tissue, delivery via a reservoir-catheter placed intraoperatively such as an Ommaya, or low-flow microinfusion for convection-enhanced delivery (CED). While these methods are advantageous in bypassing the BBB, achieving adequate drug distribution within the brain parenchyma remains a significant hurdle in approaches relying on simple diffusion, resulting in only a small volume of tissue surrounding the drug source being effectively exposed to the therapeutic agent [17-19]. Intratumoral therapy that does not rely on an infusate, such as the Gliadel wafer, which is discussed below, obviates the need for a complex delivery system but again depends on local diffusion into the surrounding tissue.

Diffusion of a compound in a tissue is dictated by its free concentration gradient and the molecular weight of the compound, being slower for higher molecular weights. In contrast to diffusion, fluid convection - or bulk flow - occurs as a result of pressure gradients and produces greater movement of molecules through tissue [20]. The concept of maintaining a pressure gradient during intracerebral drug delivery is the basis of CED-based drug delivery. CED was first investigated in a preclinical cat model by Bobo and colleagues in 1994, resulting in significantly improved distribution of labeled transferrin and sucrose as compared with simple diffusion [21], setting the stage for its subsequent utilization in a number of clinical trials in the following two decades. In CED, one or more microcatheters are placed stereotactically into the target brain parenchyma peritumorally or intratumorally. Through these catheters an infusate is actively pumped, maintaining a pressure gradient over multiple hours. Through many clinical trials using CED for intratumoral infusion therapy, it has increasingly emerged as an effective option for delivery of intracranial therapeutics, with significantly greater volume of distribution versus standard diffusion-dependent delivery methods $[5,22,23]$.

While CED has become one of the most common methods for intratumoral delivery of biologic therapies, it is not without its challenges. Troubleshooting and understanding these challenges has been an active process, as this technology is utilized in clinical trials to deliver various therapeutics intratumorally from chemotherapy to immunotoxins to gene therapy vectors. Sampson et al. [24] mixed radiolabeled albumin with a fusion product of IL-13 and mutated Pseudomonas endotoxin (PE), IL13-PE38QQR (cintredekin besudotox), to track the immunotoxin's distribution within the brain on magnetic resonance spectroscopy during CED as well as its relationship to the surgically placed catheters. They found that drug volume distribution was heavily influenced by features of catheter position, which in some cases resulted in subarachnoid leakage, ventricular leakage, back flow or extracranial leakage, all of which correlated with reduced volume distribution. Another study using an EGFR-targeted toxin with CED found leakage significantly limiting intraparenchymal volume distribution in $80 \%$ of patients [25]. Based on these observations, recommendations were made regarding technical placement of CED catheters such as maintaining a distance between catheter tip and the pial surface as well as between the catheter and the resection cavity, avoiding pial perforation, placing the catheter as deep as is safely possible, and avoiding previous catheter tracts [24]. As both the technology and surgical technique continue to be refined for improved outcomes, CED has increasingly 
become a preferred technique for effective intratumoral therapeutic delivery during clinical trials as it holds the potential to minimize interpatient confounding from variations in actual target site drug distribution.

\section{The immune system \& malignancy}

The immune system has been recognized as playing an important role in malignancy surveillance and prevention throughout the body [26,27]. The complex pathophysiology of the immune system's role in malignancy is beyond the scope of this review, and is discussed elsewhere [28]; however, discussion of basic underlying immunological principles relating to current intratumoral therapies for HGGs is necessary.

As the development and progression of malignancy represents a failure of anticancer immune surveillance, many intratumoral therapies involve a mechanism of action that aims to amplify, stimulate or otherwise target the immune system [29]. The immune system is classically divided into two broad categories consisting of innate immunity and adaptive immunity. The innate immune system includes a cellular surveillance system of macrophages, monocytes, neutrophils, natural killer cells and dendritic cells, capable of phagocytosis and releasing of cytokines and chemokines to stimulate an immune response. This cellular surveillance system relies on distinguishing normal cells from abnormal cells and foreign material through recognition of the molecular pattern of surface markers, or antigens. Malignant cells may produce abnormal antigens, tumor antigens encoded by tumor-specific mutated genes (called 'neoantigens'), or produce products associated with cell death or damage, deemed danger-associated molecular patterns, either of which may be recognized as abnormal by dendritic cells thereby eliciting an antitumor immune response $[28,30]$. Intratumoral immunostimulatory therapy being clinically evaluated uses oligodeoxynucleotides as a powerful immune stimulant [31,32].

In addition to stimulating the immune system to trigger or upregulate its recognition of malignant cells, another type of immune therapy, called passive immunotherapy, seeks to selectively target tumor cells with cytotoxins or radioactive compounds without specifically inducing the host immune response. Therapeutics in this category include immunotoxins and radioimmunotherapy. Immunotoxins consist of a modified cytotoxic protein, such as Pseudomonas exotoxin or diphtheria toxin, that is conjugated to a ligand or antibody for which its receptor or target is known to be either uniquely expressed or vastly overexpressed by the target tumor cells (tumor-associated antigens) relative to normal brain tissue, resulting in selective targeting of malignant cells with lowered collateral toxicity [33]. Targets for intratumorral immunotoxin therapy that have been clinically investigated include transferrin receptor, IL-4, IL-13, EGFR, and its mutant variant (EGFRvIII) [34] among others.

\section{Immunotoxins}

Immunotoxins represent a novel class of specific anticancer agents that selectively target receptors overexpressed by tumor cells. Therapeutics in this category consist of a tumor-selective ligand conjugated to a peptide protein that is a modified toxin representing a 'Trojan horse' payload being targeted for delivery to tumor cells. Pseudomonas exotoxin A and diphtheria toxin are among the most common toxins used in intratumoral therapy for HGGs. Both represent class A-B toxins that require receptor-mediated cellular uptake for activity [35]. Once inside the cell these toxins disrupt protein synthesis leading to cell death. This two-part construct allows for specific tumor cell targeting while minimizing toxin activity against normal tissue. Additionally, this mechanism of action does not depend on the rate of cell division, which is the major limitation of chemotherapy and radiation, and therefore has the potential to better target slowly dividing cancer stem cells that are increasingly being understood as drivers of treatment resistance and tumor recurrence. Coupling the specificity of immunotoxins with direct intratumoral delivery (e.g., CED) may further optimize exposure of the tumor cells to the therapeutic agent by bypassing the BBB and limiting systemic toxicity [36]. Several immunotoxins have been developed and clinically tested, as summarized in Table 1 and discussed below. Figure 1 illustrates the recombinant fusion products that have been evaluated for targeted immunotherapy; these generally consist of mutated cytotoxins conjugated to peptides. This conjugation promotes specificity by targeting the cytotoxins to receptors that are highly expressed by tumor cells. Table 1 also includes a summary of the most common adverse effects reported in these trials but is not intended to be all inclusive.

Tf-CRM107 is one of the first immunotoxins to be clinically evaluated, consisting of transferrin conjugated to diphtheria toxin with a point mutation rendering it unable to bind mammalian cells (Figure 1) [37]. Weaver and Laske [38] published the results of a multicenter Phase II trial evaluating Tf-CRM107 in the treatment of progressive or recurrent glioblastoma and anaplastic astrocytoma using CED with a primary end point of $>50 \%$ radiographic response within 12 months. Of 34 evaluable, 32\% (11) were found to have a partial or complete response on 


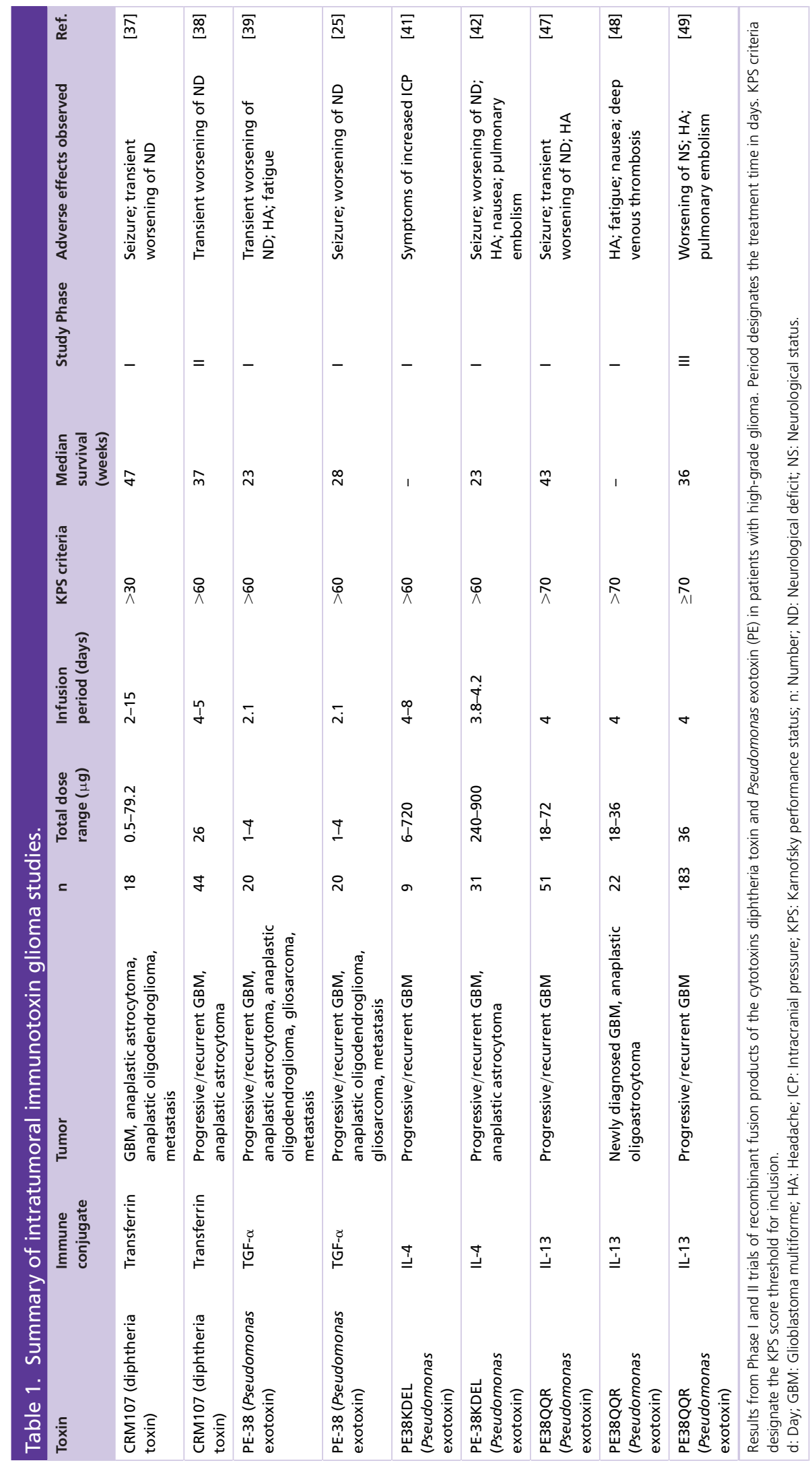




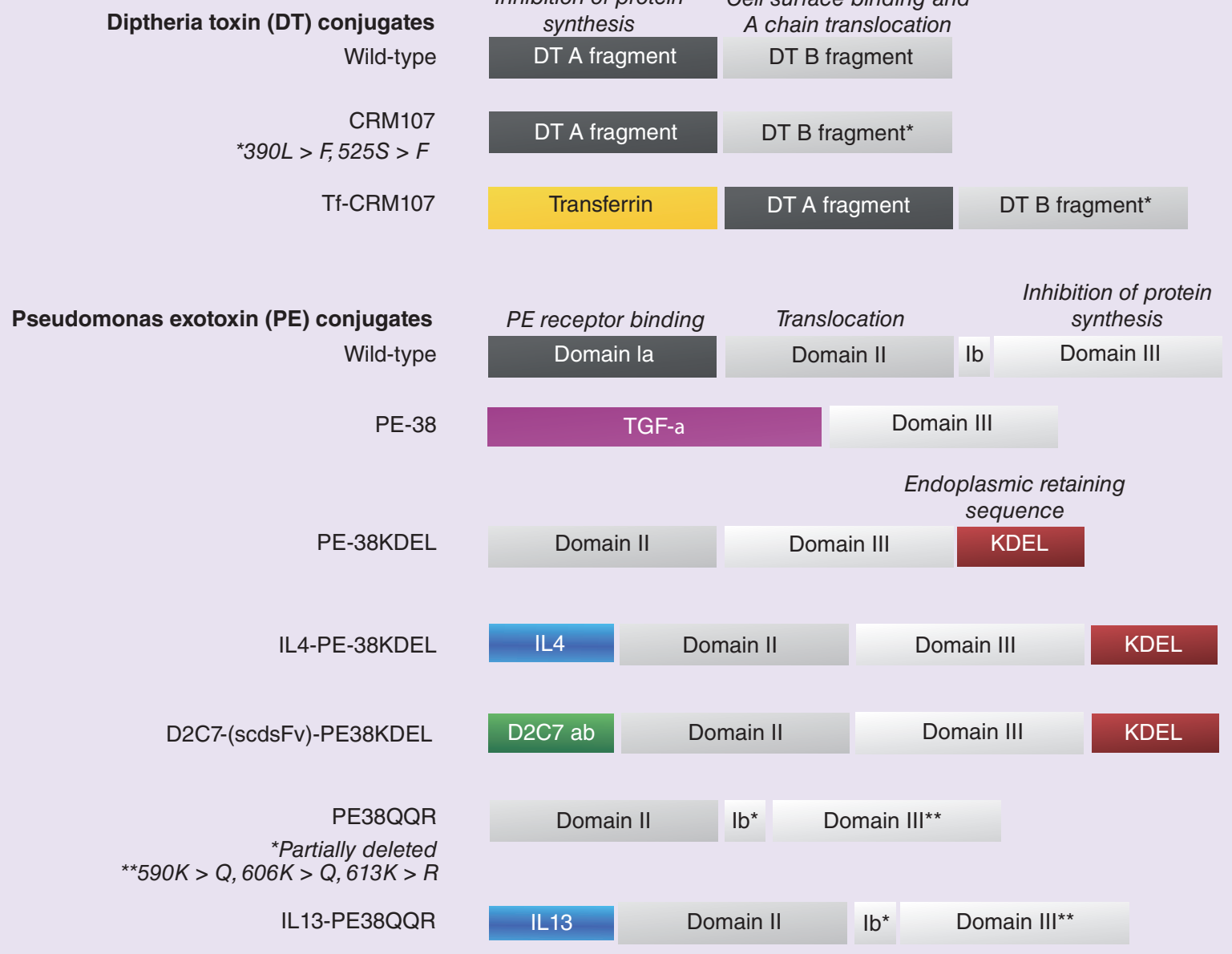

Figure 1. Conjugated immunotoxins under evaluation for the treatment of high-grade glioma. Two major classes of candidate therapies include recombinant peptide conjugates of the cytotoxins diphtheria toxin and PE derivatives. The leading candidates include fusion products of CRM107 and PE38. Tf-CRM107 is a transferrin conjugate of CRM107, a diphtheria toxin derivative, which targets the transferrin receptor. CRM107 includes a mutated B fragment (marked by *). PE derivatives include those with deletions of domain Ib, partial deletions of domain $1 \mathrm{~b}$ (marked by *), or mutations of domain III (marked by **). These PE derivatives have thus far been conjugated to TGF- $\alpha$, an endoplasmic retaining sequence (KDEL), IL-4, IL-13, D2C7-(scdsFv)-PE38KDEL, or combinations of these. PE: Pseudomonas exotoxin.

imaging and overall median survival was 9.3 months. An association between radiographic response and improved survival was also observed, suggesting a clinically significant response in these patients.

A similar Phase I study using Tf-CRM107 in children has also been completed (NCT00052624). Additionally, a Phase III trial comparing Tf-CRM107 treatment for progressive/recurrent nonresectable glioblastoma with standard-of-care chemotherapeutics (either nitrosureas, platinum compounds, temozolomide, procarbazine or PCV [procarbazine, lomustine, and vincristine]) has been completed with results yet to be released (NCT00088400). This study also evaluated possible differences in treatment efficacy associated with varying degrees of transferrin receptor expression in tumor tissue as well as serum antidiphtheria toxin antibody titers. It is not clear why the results of these studies remain unpublished despite extended intervals since completion, but this highlights an important issue regarding the need for publication of all human clinical trial results even if primary outcomes were unsuccessful, as much can be gained from learning from the experiences with these agents and delivery approaches.

$\mathrm{PE}$ has been heavily explored and clinically evaluated as an immunotoxin due to the selective but powerfully cytotoxic properties of its modified forms. Three main forms of PE have been used in clinical trials: PE-38, PE38KDEL and PE38QQR (Figure 1). The normal form of PE is a 66,000-Da protein with three domains. Domain Ia (amino acids [aa] 1-252) binds the PE receptor ubiquitous on animal cells; the function of domain 
Ib (aa365-399) remains unknown. Domain II (aa 253-364) mediates translocation of the carboxy terminal toxin fragment into the cytosol via proteolytic cleavage. Finally, domain III (aa 400-613) contains the adenosine 5'diphosphate ribosylating activity that inactivates elongation Factor 2, causing disruption of protein synthesis [39]. With the understanding of PE's molecular biology, various modifications have been made to optimize its potential as an anticancer immunotoxin.

The PE-38 form results from deletion of the Ia/Ib domains leading to intact ADP ribosylating activity but inability to bind cells, neutralizing its natural toxicity. Replacing the deleted domain with TGF- $\alpha$ yields the immunotoxin TP-38, which selectively targets cells expressing EGFR [39]. A Phase I trial was completed by Sampson et al. [25] using TP-38 delivered via CED for treatment of recurrent malignant brain tumors (17 glioblastoma, 1 gliosarcoma, 1 anaplastic oligodendroglioma, 1 metastatic spindle cell). Dose-limiting toxicity occurred in two patients (10\%) who developed grade 3 hemiparesis and another with severe fatigue. The latter was found to have intraventricular infusate leakage, which was thought to have contributed to his constitutional symptoms and both patients had residual tumor. Five patients, all with a prior known history of seizures, had a seizure during the TP-38 infusion, which was subsequently controlled with medication adjustment alone. Overall 2 of 15 patients with residual tumor at the time of treatment showed a radiographic response. Median time to progression was 14.9 weeks (range: $<1-211$ weeks) and median survival 28 weeks (range: 1.1->260 weeks). Both were greater in patients without residual disease at time of treatment, but the study was not powered to determine significance of this association. Two long-term survivors occurred at $>211$ and $>260$ weeks from TP-38 therapy. Overall the TP-38 therapy appeared to be well tolerated with a small proportion of patients showing radiographic response as well as long-term survival. Subsequently a Phase II trial for TP-38 treatment with CED in recurrent glioblastoma has been completed that will more closely evaluate time to progression, safety and survival with results still pending (NCT00104091).

A second form of PE, which has been used in clinical trials for HGGs, is PE38KDEL. Similar to PE-38, the Ia/Ib domains are deleted; however, in this case $4 \mathrm{KDEL}$, an endoplasmic retaining sequence, is conjugated to the third domain. This modification has been shown to enhance binding affinity in preclinical studies [40]. There are two tumor receptor targets that have been trialed using this toxin: IL4-PE38KDEL and D2C7-(scdsFv)-PE38KDEL. First, IL4-PE38KDEL was evaluated by Rand et al. [41] in a Phase I trial that included nine patients with progressive or recurrent glioblastoma using CED. In this dose-escalation study patients received 6-720 $\mu \mathrm{g}$ over a period of 4-8 days. The dosages were selected based on preclinical studies, starting at $0.2 \mu \mathrm{g} / \mathrm{ml}$ and escalated one log and $1.5 \operatorname{logs}$ in the second and third cohorts, respectively, up to a maximum of $6 \mu \mathrm{g} / \mathrm{ml}$; the volume infused was based on tumor volume measured on MRI. Cerebral edema was a common complication with $78 \%$ (seven out of nine) of patients developing subacute (days 10-27) or delayed (days 43-97) cerebral edema after the infusion leading to elevated intracranial pressure uncontrollable with medical therapy alone, for which they underwent a craniotomy with tumor resection. Other adverse events observed included hydrocephalus requiring cerebrospinal fluid shunt placement in two patients and weakness in two patients. There did not appear to be a significant association between dose level and occurrence of adverse events in this small population. All patients showed some radiographic response with slight reduction in thickness of enhancing portion of tumor on MRI at 4-9 days post-treatment and 66\% (six out of nine) showed interval development of tumor necrosis post-treatment MRI at 7 days to 15 months. In no case was treatment associated with necrosis of normal brain tissue. Survival and time to progression data are not given; however, one instance of long-term survival occurred in a 71-year-old male who had already undergone resection and radiation twice prior to IL4-PE38KDEL treatment. He received a total of $360 \mu \mathrm{g}$ over 5 days via two catheters. This patient showed shrinkage of tumor on serial MRIs and hypometabolism on PET without recurrence at 15 months and survival of $>18$ months.

A second, multicenter Phase I study using IL4-PE38KDEL with CED in patients with recurrent/progressive glioblastoma or anaplastic astrocytomas included 31 patients, 25 with glioblastoma [42]. In this dose-escalation study, the starting dose was $6 \mu \mathrm{g} / \mathrm{ml}(40 \mathrm{ml})$ based on the maximum administered dose in the study above and increasing up to $9 \mu \mathrm{g} / \mathrm{ml}(100 \mathrm{ml})$ in three steps after surveilling for dose-limiting toxicity. All patients reported some form of adverse event with the most common being seizures (84\%), headache (45\%), weakness (32\%) and cerebral edema (32\%). No clear dose-related trends were noted to correlate with adverse events. In no case was neurosurgical intervention such as craniotomy or permanent cerebrospinal fluid diversion required to manage adverse neurological events. Most patients showed a distinct region of decreased contrast enhancement suggestive of tumor necrosis with subsequent increase in contrast enhancement at 4 weeks that then gradually tapered over several weeks. Overall median survival was 8.2 and 5.8 months in the glioblastoma group. There was at least 
one case of long-term survival at $>3$ years in a 43-year-old male with right frontal glioblastoma who underwent resection of residual tumor and necrotic tissue 3 months after treatment.

Overall IL4-PE38KDEL initially showed a high rate of adverse events in the form or cerebral edema in the first clinical trial above, leading to the majority of patients requiring neurosurgical intervention. This phenomenon was largely eliminated in the second trial; however, the rate of adverse events remained high but less severe and more acceptable. In either study there did not appear strong dose-related trends with the occurrence of adverse events. IL4-PE38KDEL is being further investigated in Phase II trials. One, which is completed with results pending, will further evaluate the safety and tolerability of the immunotoxin treatment via CED in recurrent glioblastoma followed by surgical resection 3 weeks later (NCT00014677). Another Phase II trial that is currently recruiting will evaluate efficacy and safety of IL4-PE38KDEL treatment in recurrent glioblastoma via CED with a goal of 43 patients.

The second immunotoxin using PE38KDEL has the single-chain variable-region antibody fragment of the D2C7 monoclonal antibody fused to domains II and III of PE38KDEL. The D2C7 antibody is highly reactive against both wild-type and variant III mutant EGFRs allowing D2C7-(scdsFv)-PE38KDEL to selectively bind both types of EGFR, which is commonly overexpressed and amplified in HGG cells [43,44]. Currently a Phase I trial is recruiting to evaluate the use of D2C7-(scdsFv)-PE38KDEL treatment via CED in patients with recurrent HGG (NCT02303678).

A third form of PE is PE38QQR, which is a modified version of PE38 to optimize its toxicity. Specifically, PE38-based immunotoxins were initially synthesized using heterobifunctional cross-linking reagents, which attach the target ligand to the N-terminal methionine or lysine residues of the toxin. Because domain III contains three lysine residues at positions 590, 606 and 613 cross linking may occur at these locations which can interfere with the toxin's translocation into the cytosol reducing its effect, whereas binding of the ligand at the $\mathrm{N}$-terminal methionine maintains this function [45,46]. With this understanding, PE38 was modified replacing the three lysine residues on domain III with glutamine and arginine as well as adding a peptide containing a lysine residue to the $\mathrm{N}$-terminus. This allows for a more homogeneous generation of the immunotoxin with all ligands coupled to the $\mathrm{N}$-terminus, optimizing uptake of the toxin by the target cells producing enhanced intracellular retention as well as reduced hepatotoxicity. The result is PE38QQR, which has been shown in initial preclinical studies to have better synthetic yield and improved cytotoxicity to target cells [46].

PE38QQR conjugated to IL-13 (IL13-PE38QQR) has been extensively investigated in clinical studies. In an aggregate report on three Phase I trials, Kunwar et al. [47] evaluated the safety and tolerability of IL13PE38QQR treatment for 51 patients with recurrent or progressive malignant gliomas ( $90 \%$ glioblastoma) via CED administered 1-3 days after tumor resection. Catheter placement targeted either residual enhancement or fluid-attenuated inversion recovery (FLAIR) signal concerning for microscopic disease around the resection cavity on MRI. Treatment was infused over $96 \mathrm{~h}$ in escalating dosages ranging from 18 to $72 \mu \mathrm{g}$. Dose-limiting toxicities occurred in two of three patients receiving the highest dosage who developed cerebral edema, one of which required surgical decompression. Given this, the acceptable maximum dose was determined to be $36 \mu \mathrm{g}$ $(0.5 \mu \mathrm{g} / \mathrm{ml}$ at $0.75 \mathrm{ml} / \mathrm{h})$. The most common adverse events were headache $(41 \%)$, sensory disturbance $(25 \%)$ and aphasia (18\%) with no relationship between drug concentration and severity of adverse events. Median survival for patients with glioblastoma was 42.7 weeks with a 1-year survival of $39.1 \%$. Patients with optimally placed catheters according to guidelines discussed above were found to have significantly greater survival at 55.6 weeks than patients with suboptimal placement of one or both catheters at 37.4 weeks. Progression-free survival at 1 and 2 years occurred in 17.6 and 13.7\%, respectively. Overall the IL13-PE38QQR treatment demonstrated an acceptable safety profile and survival compared favorably for patients with glioblastoma relative to established intratumoral therapy for recurrent glioblastoma. Particularly the 1- and 2-year survival appeared to be comparable or greater than that of bis-chloroethyl nitrosurea (Gliadel wafer; Guilford Pharmaceuticals, MD, USA) wafers, at $\leq 15 \%$ and $\leq 10 \%$, respectively, with several patients with recurrent glioblastoma having prolonged progression-free survival.

In another Phase I trial, Vogelbaum et al. [48] evaluated the safety of IL13-PE38QQR treatment via CED in 22 newly diagnosed malignant gliomas (95\% glioblastoma) after gross total resection in conjunction with standard adjuvant therapy of external beam radiation therapy with or without temozolomide. Guided by the dosage data from Kunwar $e t a l$. [47], a dose escalation regimen was used ranging from 18 to $36 \mu \mathrm{g}$ delivered over $96 \mathrm{~h}$. Two doselimiting toxicities occurred out of the 16 patients receiving the highest dose with one developing status epilepticus at 10 weeks and the other with expressive dysphasia at 1 week. The most common adverse events included fatigue (73\%), headache (50\%) and nausea (41\%); no relationship was observed between dosage and adverse event severity. 
Survival was $50 \%$ after a median follow-up of 44 weeks and ranged from 5 to 113 weeks. Progression-free survival at 40 weeks was $27 \%$. This study further supported the safety and tolerability of IL13-PE38QQR treatment when used in series with standard therapy.

Given the promising results of the initial studies, Kunwar et al. continued with a Phase III trial (PRECISE) directly comparing IL13-PE38QQR with Gliadel wafers, the only intratumoral chemotherapeutic approved by the US FDA [49]. This multicenter trial randomized 296 patients with recurrent glioblastoma in a 2:1 ratio to receive either postoperative IL13-PE38QQR via CED or Gliadel wafers (GW). Similar to the above, catheter placement was directed at regions with residual contrast enhancement or FLAIR abnormality on MRI. IL13-PE38QQR infusion began 3-8 days after resection and took place over $96 \mathrm{~h}$ for a total dose of $36 \mu \mathrm{g}$. Safety profiles were similar in both groups and there was no significant difference in median survival between treatment arms at 45.3 and 39.8 weeks for IL13-PE38QQR and GW, respectively. Catheter placement, however, was noted to be a challenge with only $68 \%$ of catheters placed optimally although this did not significantly affect survival on post hoc analysis. This study effectively demonstrated noninferiority of IL13-PE38QQR versus GW for recurrent GBM, and this treatment is pending further development and Federal Drug Administration approval for use as an adjuvant therapy. While this study tracked catheter position, it did not specifically track distribution of the immunotoxin infusate, which is thought to be a critical determination of efficacy. Therefore, it is possible these results represent an underestimate of the true impact of IL13-PE38QQR if ideal distribution was to be achieved and verified in each case.

Immunotoxin therapy for HGG remains an advancing and promising modality for adjuvant treatment in this disease. However, there has yet to be any single agent or trial that has demonstrated a profound step forward in survival, and technical challenges still exist for both drug delivery and side-effect management. The response rates of the various compounds discussed above can be significantly variable but seem to share certain general characteristics including a pattern of better treatment response if minimal or no residual tumor is present at time of treatment initiation and optimal tissue distribution is achieved. Side effects including neurological symptoms tend to be common but are also transient in most cases. Despite these challenges, immunotoxin therapy continues to be a growing area of $R \& D$ as these therapeutics are further refined in parallel with advancements in understanding HGG tumor biology to better improve target specificity as well as optimize drug delivery, while minimizing collateral effects on normal tissue.

\section{Conclusion}

Immunotoxin therapy for HGG continues to show promise as developments are made in drug specificity in parallel with enhanced understanding of tumor cell biology. Additionally, technical advancements like optimization of CED have the potential to improve the reliability of therapeutic delivery and limit target drug bioavailability confounding in future clinical trials. Future research must seek to optimize both therapeutic action at the target site and exposure of the tumor cells to the therapeutic agent through better understanding of drug and cell distribution. Targeted intratumoral therapies hold great promise as an increasingly important new paradigm in the design of HGG clinical trials as new research continues to be transitioned from bench to bedside.

\section{Future perspective}

Biological intratumoral therapies represent an advancing adjuvant treatment option for high-grade gliomas. While no single therapeutic immunotoxin has produced results profound or consistent enough to establish it as a standard of care, results are promising as improvements are made in methods of technical delivery and improved toxin targeting. Given the widely variable range of outcomes observed in studies to date, moving forward it will be paramount to better understand the underlying patient-specific factors that influence the degree to which treatment response is seen. These may include tumor genetics and epigenetics, technique of delivery, and the patient's immune status among other factors that can affect tumor response and patient tolerance. Characterizing these variables is key to being able to parse out those patients where maximal treatment benefit can be achieved with optimal reliability. As the field grows and evolves this may manifest as designed patient-specific treatment pathways customized based on tumor genetic expression profiles and patient characteristics.

Financial \& competing interests disclosure

The authors have no relevant affiliations or financial involvement with any organization or entity with a financial interest in or financial conflict with the subject matter or materials discussed in the manuscript. This includes employment, consultancies, honoraria, stock ownership or options, expert testimony, grants or patents received or pending, or royalties.

No writing assistance was utilized in the production of this manuscript. 
Open access

This work is licensed under the Attribution-NonCommercial-NoDerivatives 4.0 Unported License. To view a copy of this license, visit http://creativecommons.org/licenses/by-nc-nd/4.0/

\section{Executive summary}

Background

- Multimodal therapeutic approaches are necessary to treat high-grade gliomas (HGG).

- Surgically delivered biological therapies provide a novel solution to longstanding pharmacokinetic problems (e.g., blood-brain barrier efflux pumps) and pharmacological problems (e.g., specificity, toxicity) in HGG treatment.

Intratumoral therapeutic delivery

- Intratumoral delivery permits direct-to-target delivery of biologics that would traditionally be poor pharmacokinetic candidates for HGG.

- Direct infusion into the brain parenchyma using stereotactically placed microcatheters is currently the most common route of administration for intratumoral delivery in HGG.

The immune system \& malignancy

- The immune system has been recognized as playing an important role in combating malignancy.

- Biological therapies that activate the immune response have been in use for HGG since the 1970s.

- Immune-modulating therapeutics may be administered via intratumoral delivery to facilitate more efficient activation of the immune response.

Immunotoxins

- Immunotoxins provide a 'Trojan horse' approach to treatment.

- Current therapies consist of cellular toxins conjugated to modified peptides that target receptors selectively overexpressed by cancer cells.

- While many advancements have been made, clinical trial results remain mixed. A major technical limitation is achieving ideal volume of distribution for experimental therapeutics.

\section{Conclusion}

- Immunotoxin-based therapies may provide a major advancement in HGG therapy as they continue to be developed.

- Future studies will evaluate the efficacy of these agents in clinical trials and improve candidate therapies' delivery, specificity and efficacy.

\section{References}

1. Omuro A, DeAngelis LM. Glioblastoma and other malignant gliomas: a clinical review. JAMA 310(17), 1842-1850 (2013).

2. Raizer JJ, Fitzner KA, Jacobs DI et al. Economics of malignant gliomas: a critical review. J. Oncol. Pract. 11(1), e59-e65 (2015).

3. Sachs E. The treatment of glioblastomas with radium. J. Neurosurg. 11(2), 119-121 (1954).

4. Gabathuler R. Approaches to transport therapeutic drugs across the blood-brain barrier to treat brain diseases. Neurobiol. Dis. 37(1), 48-57 (2010).

5. Vandergrift WA, Patel SJ, Nicholas JS, Varma AK. Convection-enhanced delivery of immunotoxins and radioisotopes for treatment of malignant gliomas. Neurosurg. Focus. 20(4), E13 (2006).

6. Gorer PA. Some recent work on tumor immunity. Adv. Cancer. Res. 4, 149-186 (1956).

7. Hauschka TS. Immunologic aspects of cancer: a review. Cancer. Res. 12(9), 615-633 (1952).

8. Grace JTJ Jr, Perese DM, Metzgar RS, Sasabe T, Holdridge B. Tumor autograft responses in patients with glioblastoma multiforme. J. Neurosurg. 18(2), 159-167 (1961).

9. Ridley A, Cavanagh JB. Lymphocytic infiltration in gliomas: evidence of possible host resistance. Brain 94(1), 117-124 (1971).

10. Brooks WH, Markesbery WR, Gupta GD, Roszman TL. Relationship of lymphocyte invasion and survival of brain tumor patients. Ann. Neurol. 4(3), 219-224 (1978).

11. Di Lorenzo N, Palma L, Nicole S. Lymphocytic infiltration in long-survival glioblastomas: possible host's resistance. Acta Neurochirurgica 39(1), 27-33 (1977).

12. Palma L, Di Lorenzo N, Guidetti B. Lymphocytic infiltrates in primary glioblastomas and recidivous gliomas. Incidence, fate, and relevance to prognosis in 228 operated cases. J. Neurosurg. 49(6), 854-861 (1978).

13. Young H, Kaplan A, Regelson W. Immunotherapy with autologous white cell infusions ('lymphocytes') in the treatment of recurrrent glioblastoma multiforme: a preliminary report. Cancer 40(3), 1037-1044 (1977). 
14. Steinbok P, Thomas JP, Grossman L, Dolman CL. Intratumoral autologous mononuclear cells in the treatment of recurrent glioblastoma multiforme. A Phase I (toxicity) study. J. Neurooncol. 2(2), 147-151 (1984).

15. Jacobs SK, Wilson DJ, Kornblith PL, Grimm EA. Interleukin-2 or autologous lymphokine-activated killer cell treatment of malignant glioma: Phase I trial. Cancer. Res. 46 (4 Pt 2), 2101-2104 (1986).

16. Abbott NJ, Ronnback L, Hansson E. Astrocyte-endothelial interactions at the blood-brain barrier. Nat. Rev. Neurosci. 7(1), 41-53 (2006).

17. Kroin JS, Penn RD. Intracerebral chemotherapy: chronic microinfusion of cisplatin. Neurosurgery 10(3), 349-354 (1982).

18. Garfield J, Dayan AD. Postoperative intracavitary chemotherapy of malignant gliomas. A preliminary study using methotrexate. J. Neurosurg. 39(3), 315-322 (1973).

19. Lee Sendelbeck S, Urquhart J. Spatial distribution of dopamine, methotrexate and antipyrine during continuous intracerebral microperfusion. Brain Res. 328(2), 251-258 (1985).

20. Cserr HF, Ostrach LH. Bulk flow of interstitial fluid after intracranial injection of blue dextran 2000. Exp. Neurol. 45(1), 50-60 (1974).

21. Bobo RH, Laske DW, Akbasak A, Morrison PF, Dedrick RL, Oldfield EH. Convection-enhanced delivery of macromolecules in the brain. Proc. Natl Acad. Sci. USA 91(6), 2076-2080 (1994).

22. Lieberman DM, Laske DW, Morrison PF, Bankiewicz KS, Oldfield EH. Convection-enhanced distribution of large molecules in gray matter during interstitial drug infusion. J. Neurosurg. 82(6), 1021-1029 (1995).

23. Sampson JH, Akabani G, Friedman AH et al. Comparison of intratumoral bolus injection and convection-enhanced delivery of radiolabeled antitenascin monoclonal antibodies. Neurosurg. Focus 20(4), E14 (2006).

24. Sampson JH, Brady ML, Petry NA et al. Intracerebral infusate distribution by convection-enhanced delivery in humans with malignant gliomas: descriptive effects of target anatomy and catheter positioning. Neurosurgery 60(2 Suppl 1), ONS89-98; discussion ONS98-89 (2007).

25. Sampson JH, Akabani G, Archer GE et al. Intracerebral infusion of an EGFR-targeted toxin in recurrent malignant brain tumors. Neuro. Oncol. 10(3), 320-329 (2008).

26. Makkouk A, Weiner GJ. Cancer immunotherapy and breaking immune tolerance: new approaches to an old challenge. Cancer Res. 75(1), 5-10 (2015).

27. Swann JB, Smyth MJ. Immune surveillance of tumors. J. Clin. Investig. 117(5), 1137-1146 (2007).

28. McGranahan T, Li G, Nagpal S. History and current state of immunotherapy in glioma and brain metastasis. Ther. Adv. Med. Oncol. 9(5), 347-368 (2017).

29. Gajewski TF, Schreiber H, Fu YX. Innate and adaptive immune cells in the tumor microenvironment. Nat. Immunol. 14(10), 1014-1022 (2013).

30. Gotwals P, Cameron S, Cipolletta D et al. Prospects for combining targeted and conventional cancer therapy with immunotherapy. Nat. Rev. Cancer 17(5), 286-301 (2017).

31. Carpentier A, Laigle-Donadey F, Zohar S et al. Phase I trial of a CpG oligodeoxynucleotide for patients with recurrent glioblastoma. Neuro. Oncol. 8(1), 60-66 (2006).

32. Carpentier A, Metellus P, Ursu R et al. Intracerebral administration of CpG oligonucleotide for patients with recurrent glioblastoma: a Phase II study. Neuro. Oncol. 12(4), 401-408 (2010).

33. Daga A, Bottino C, Castriconi R, Gangemi R, Ferrini S. New perspectives in glioma immunotherapy. Curr. Pharm. Des. 17(23), 2439-2467 (2011).

34. Chandramohan V, Sampson JH, Pastan I, Bigner DD. Toxin-based targeted therapy for malignant brain tumors. Clin. Dev. Immunol. 2012, 480429 (2012).

35. Weldon JE, Pastan I. A guide to taming a toxin-recombinant immunotoxins constructed from Pseudomonas exotoxin A for the treatment of cancer. FEBS J. 278(23), 4683-4700 (2011).

36. Rainov NG, Gorbatyuk K, Heidecke V. Clinical trials with intracerebral convection-enhanced delivery of targeted toxins in malignant glioma. Rev. Recent Clin. Trials 3(1), 2-9 (2008).

37. Laske DW, Youle RJ, Oldfield EH. Tumor regression with regional distribution of the targeted toxin TF-CRM107 in patients with malignant brain tumors. Nat. Med. 3(12), 1362-1368 (1997).

38. Weaver M, Laske DW. Transferrin receptor ligand-targeted toxin conjugate (Tf-CRM107) for therapy of malignant gliomas. J. Neurooncol. 65(1), 3-13 (2003).

39. Sampson JH, Akabani G, Archer GE et al. Progress report of a Phase I study of the intracerebral microinfusion of a recombinant chimeric protein composed of transforming growth factor (TGF)- $\alpha$ and a mutated form of the Pseudomonas exotoxin termed PE-38 (TP-38) for the treatment of malignant brain tumors. J. Neurooncol. 65(1), 27-35 (2003).

40. Puri RK, Hoon DS, Leland P et al. Preclinical development of a recombinant toxin containing circularly permuted interleukin 4 and truncated Pseudomonas exotoxin for therapy of malignant astrocytoma. Cancer Res. 56(24), 5631-5637 (1996). 
41. Rand RW, Kreitman RJ, Patronas N, Varricchio F, Pastan I, Puri RK. Intratumoral administration of recombinant circularly permuted interleukin-4-Pseudomonas exotoxin in patients with high-grade glioma. Clin. Cancer Res. 6(6), 2157-2165 (2000).

42. Weber F, Asher A, Bucholz R et al. Safety, tolerability, and tumor response of IL4-Pseudomonas exotoxin (NBI-3001) in patients with recurrent malignant glioma. J. Neurooncol. 64(1-2), 125-137 (2003).

43. Bao X, Pastan I, Bigner DD, Chandramohan V. EGFR/EGFRvIII-targeted immunotoxin therapy for the treatment of glioblastomas via convection-enhanced delivery. Receptors Clin. Investig. 3(4), e1430 (2016).

44. Chandramohan V, Bao X, Keir ST et al. Construction of an immunotoxin, D2C7-(scdsFv)-PE38KDEL, targeting EGFRwt and EGFRvIII for brain tumor therapy. Clin. Cancer Res. 19(17), 4717-4727 (2013).

45. Batra JK, Jinno Y, Chaudhary VK et al. Antitumor activity in mice of an immunotoxin made with anti-transferrin receptor and a recombinant form of Pseudomonas exotoxin. Proc. Natl Acad. Sci. USA 86(21), 8545 (1989).

46. Debinski W, Pastan I. An immunotoxin with increased activity and homogeneity produced by reducing the number of lysine residues in recombinant Pseudomonas exotoxin. Bioconjug. Chem. 5(1), 40-46 (1994).

47. Kunwar S, Prados MD, Chang SM et al. Direct intracerebral delivery of cintredekin besudotox (IL13-PE38QQR) in recurrent malignant glioma: a report by the Cintredekin Besudotox Intraparenchymal Study Group. J. Clin. Oncol. 25(7), 837-844 (2007).

48. Vogelbaum MA, Sampson JH, Kunwar S et al. Convection-enhanced delivery of cintredekin besudotox (interleukin-13-PE38QQR) followed by radiation therapy with and without temozolomide in newly diagnosed malignant gliomas: Phase I study of final safety results. Neurosurgery 61(5), 1031-1038 (2007).

49. Kunwar S, Chang S, Westphal M et al. Phase III randomized trial of CED of IL13-PE38QQR vs Gliadel wafers for recurrent glioblastoma. Neuro. Oncol. 12(8), 871-881 (2010). 